Ann. Biol. anim. Bioch. Biophys., 1979, 19 (1B), 199-205.

\title{
Influence du niveau des apports énergétiques pendant la fin de la gestation sur l'insulinémie et l'aminoacidémie des brebis et de leurs agneaux
}

par J. GRIZARD, P. PATUREAU-MIRAND, M. TISSIER *

avec la collaboration technique de Odile BERNARD, G. BAYLE, A. BRELURUT*, J. LEROUX*

Laboratoire d'éfude du Métabolisme azoté

* Laboratoire de Production ovine

I.N.R.A., Theix, Saint-Genès-Champanelle

63110 Beaumont.

Summary. Effect of energy intake during late pregnancy on plasma insulin and blood free amino acids in ewes and their lambs.

This $2 \times 2$ factorial experiment (table 1 ) was carried out in the autumn on 22 Limousine ewes during pregnancy and lactation. The animals bore or were suckling twins. The experimental treatments included 2 levels of energy intake during 7 weeks prepartum (high : $\mathrm{H}$; low : B) and 2 states of body reserves at 8 weeks prepartum (fat : $G$; lean : $M$ ). Jugular blood samples were taken from ewes at 34, 20 and 6 days prepartum, and from lambs at lambing and at 6 hrs., 24 hrs., 12 days and 24 days after lambing. Blood glucose, urea and free amino acids and plasma insulin were recorded. Reducing dietary energy supply did not change lamb birth weight in autumn (fig. 1A). It contrasted with a large diminution in the spring (Grizard ef al., 1979a, b). There was a decrease of glucose and of some non-essential free amino acids (alanine, glutamate, proline, citrulline) in the blood of the ewes. Most of the non-essential free amino acids decreased during the first day of life in the blood of lambs (fig. 2A). Reducing body reserves resulted in an increase of glucose and glutamine but in a decrease of some other free amino acids (glutamate, ornithine, histidine) in the blood of ewes (fig. 1B). It increased daily weight gain during the first 3 weeks of life in lambs (fig. 2B). At the beginning of life, plasma insulin in lambs seemed to be related to birthweight and to blood glucose in the mothers.

Chez la brebis portant plusieurs foetus, la quantité d'énergie captée par l'utérus en fin de gestation sous forme de glucose (environ $25 \mathrm{~g} \mathrm{p.j.p.} \mathrm{kg} \mathrm{de} \mathrm{fœetus)} \mathrm{(Christenson}$ ef Prior, 1978), est très élevée puisque la croissance des fœtus est très importante. En revanche, la quantité de glucose absorbée par le tube digestif est très faible et il doit être apporté en grande partie par la néoglucogenèse à partir de l'acide propionique, de l'acide lactique et des acides aminés. Pendant cette période, toute réduction de l'apport énergétique de la ration provoque alors de fortes hypoglycémies et des augmentations de l'utilisation des acides aminés à des fins énergétiques chez les brebis (Grizard ef al., 1977, 1979a, b). Celles-ci n'entraînent de réductions du poids 
des agneaux à la naissance, que dans le cas d'une alimentation hivernale (Tissier ef Thériez, 1979).

Pour préciser ces phénomènes, nous avons éfudié l'influence d'une restriction énergétique en fin de gestation et de l'absence de réserves corporelles suffisantes à l'approche du dernier tiers de la gestation, sur l'évolution de quelques composés sanguins (glucose, acides aminés libres, urée, insuline) pendant la fin de la gestation chez les brebis et de la naissance à l'âge de 24 jours chez leurs agneaux. Le présent travail a été effectué en aułomne, comme celui de Grizard et al. (1977) ; ils complètent d'autres travaux réalisés au printemps (Grizard ef al. 1979a et $b$ ).

\section{Matériel et méthodes.}

Des brebis de la race limousine âgées de 3 à 6 ans, portant puis allaitant 2 agneaux, sont réparties en 4 lots $(\mathrm{GH}, \mathrm{GB}, \mathrm{MH}$ et $\mathrm{MB})$ selon un schéma factoriel $2 \times 2$ permettant d'apprécier l'influence des apports d'énergie « haut» $(H)$ ef «bas » $(B)$ pendant les 7 dernières semaines de la gestation et de l'état « gras » $(G)$ et « maigre » $(M)$ des réserves corporelles à l'approche du dernier tiers de la gestation. Les agnelages se situent approximativement le 13 novembre.

Les brebis sont logées pendant les 7 dernières semaines de la gestation ef les 6 premières semaines de la lactation en cages individuelles sur caillebotis dans des locaux éclairés de $7 \mathrm{~h}$ à $19 \mathrm{~h}$ environ et de température comprise entre 12 et $16^{\circ} \mathrm{C}$; elles reçoivent un repas par jour distribué à 13,5 h, constitué de l'aliment concentré à base de maïs ef de tourteau de soja ( $1 / 3$ de la matière organique digestible) et de luzerne deshydratée et compactée ( $2 / 3$ de la matière organique digestible). Les apports énergétiques et azotés sont comparés aux apports estimés comme nécessaires au poids des agneaux à la naissance et aux quantités de lait produites, sur le tableau 1.

Les agneaux sont logés avec leur mère et n'ingèrent que le lait maternel pendant la période expérimentale.

Des prélèvements de sang jugulaire sont effectués chez les brebis $2,5 \mathrm{~h}$ ef $19 \mathrm{~h}$ après le repas (respectivement 10 et $50 \mathrm{ml}$ environ) à approximativement 34,20 et 6 jours prepartum. Des prélèvements de sang jugulaire (environ $5 \mathrm{ml}$ ) sont également effectués chez les agneaux à la naissance, à l'âge précis de 6 et 24 h (après avoir ingéré du colostrum) puis pendant la période d'allaitement (à l'âge moyen de 12 et 24 jours).

Le glucose et l'urée sont dosés seulement chez les brebis dans les échantillons de sang déprotéinisés par l'acide trichloracétique à 2,5 p. 100 respectivement selon les méthodes à la glucose-oxydase et la diacétyl-monoxime au moyen d'une chaîne d'analyse automatique. Les acides aminés libres des sangs groupés par lot sont dosés chez les brebis, dans les échantillons prélevés à 2,5 h après le repas et chez les agneaux. Ils sont extraits du sang total par épuisements successifs à l'éthanol à 82 p. 100 contenant 2 p. 100 de thiodiglycol ; les extraits sont purifiés sur Amberlite IR 120 (Pawlak et Pion, 1968). Les acides aminés sont séparés ef dosés sur résine échangeuse d'ions au moyen d'un analyseur automatique (Moore, Spackman et Stein, 1958). L'insuline plasmatique est dosée par radioimmunologie selon la technique du double anticorps au moyen des trousses de dosage in vitro CEA-IRE-SORIN INSIK 1 ; l'insuline standard est l'insuline humaine. 


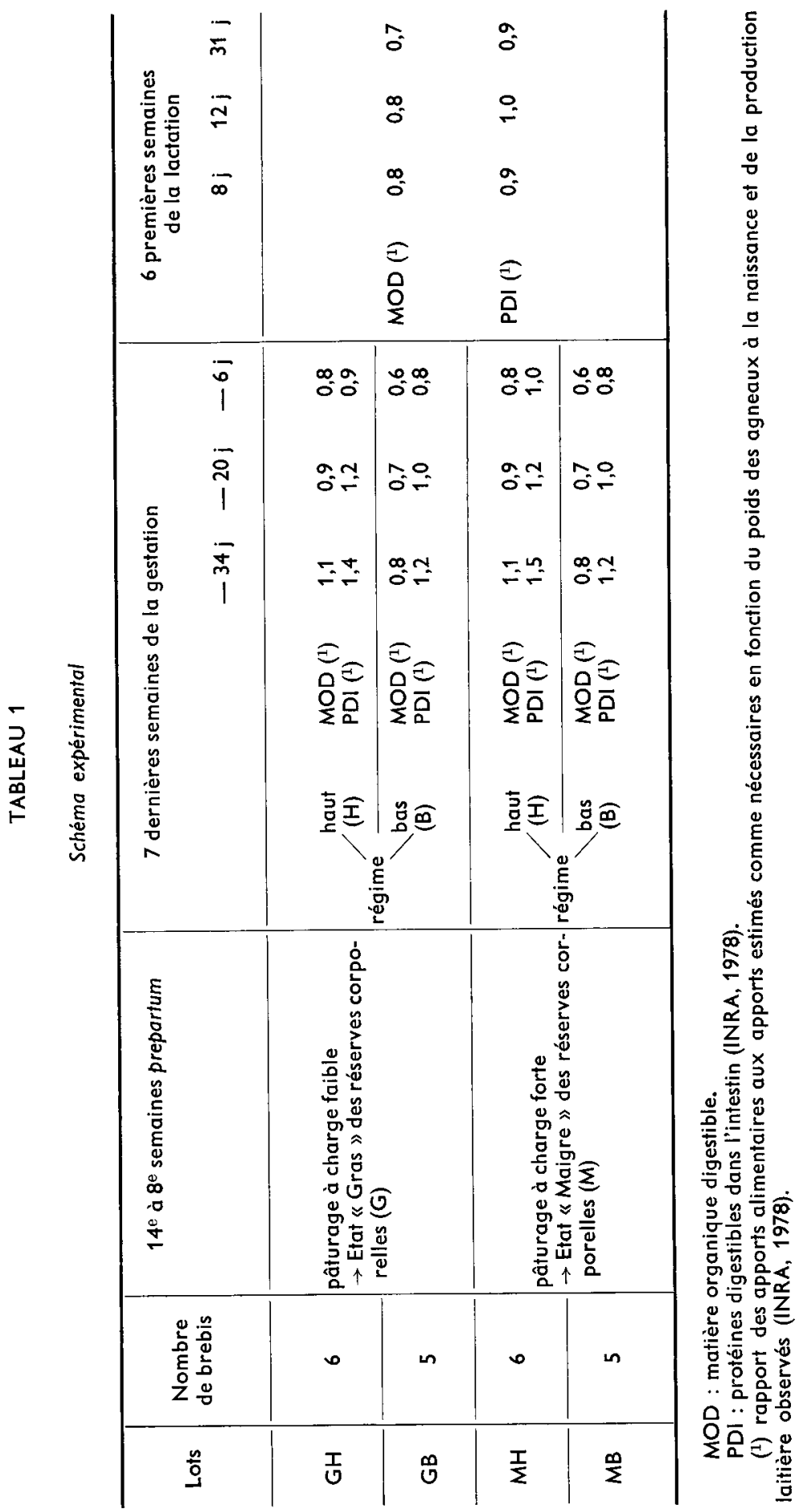




\section{Résultats et discussion.}

\section{En fin de gestation.}

La sous-alimentation énergétique n'entraîne pas de diminution du poids des agneaux à la naissance (fig. 1A) contrairement à ce qui se passe au printemps (Grizard ef al., 1979a) ; les quantités d'énergie et de matières azotées captées par l'utérus gravide ne sont donc pas modifiées alors que les apports alimentaires d'énergie et de matières azotées sont sensiblement diminués (tabl. 1). La glycémie est diminuée (fig. 1A), ce qui peut être dû à une baisse de la synthèse de glucose à partir de l'acide propionique (Prior et Christenson, 1976). Les teneurs sanguines de certains acides aminés non indispensables libres sont diminuées ef l'urémie est augmentée ; ceci traduit un accroissement du catabolisme des acides aminés et de la synthèse de glucose à partir de ces composés. En revanche, il n'y a pas de modifications notables des teneurs sanguines en acides aminés indispensables et semi-indispensables libres; ceci indique que l'augmentation du catabolisme des protéines corporelles doit compenser à la fois un catabolisme accru et un ralentissement de leurs apports par l'intestin (Grizard ef al., 1977 ; Grizard ef al., 1979a). L'insulinémie n'est pas significativement diminuée.

(A)

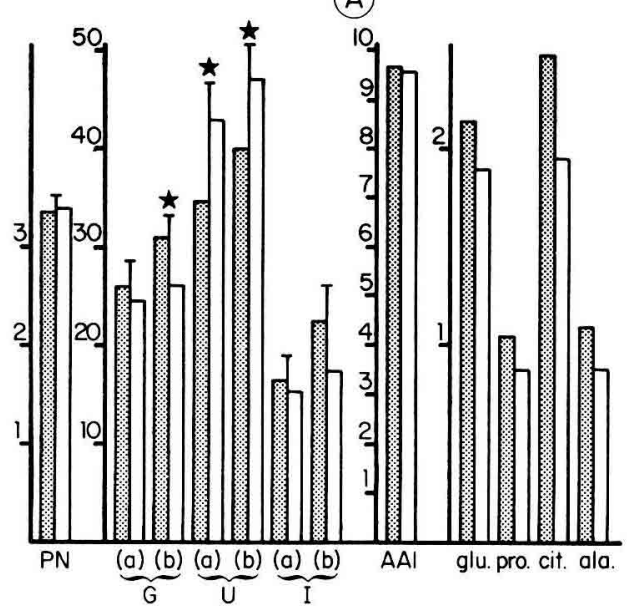

(B)

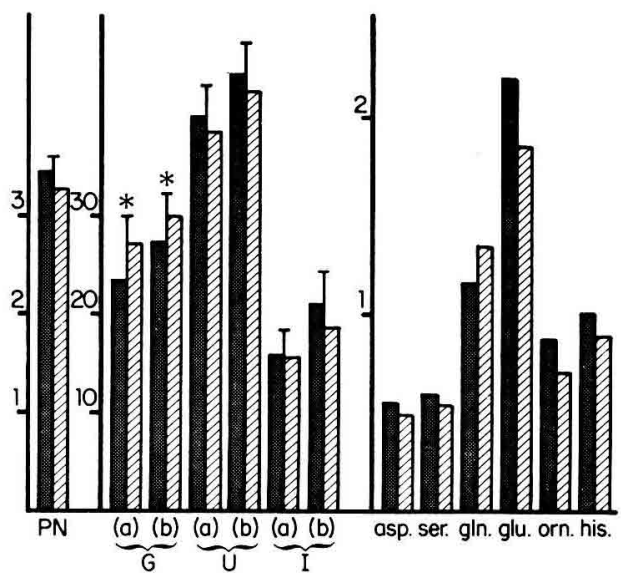

FIG. 1. - Insulinémie, glycémie, urémie ef teneurs en acides aminés libres du sang des brebis en fin de gestation (1) ef poids des agneaux à la naissance.

$\mathrm{I}$ : insuline ( $\mu \mathrm{U}$ p. $\mathrm{ml}$ de plasma) ; G : glucose (mg p. $100 \mathrm{~g}$ de sang) ; $U$ : urée (mg p. $100 \mathrm{~g}$ de sang ; AAl : somme des teneurs en acides aminés indispensables et semi-indispensables (mg p. $100 \mathrm{~g}$ de sang) ; asp, ser, gln, glu, pro, cit, ala, orn, his : acides aminés (mg p. $100 \mathrm{~g}$ de sang) ; PN : poids de naissance $(\mathrm{kg}) ;(a): 2,5$ heures après le repas ; (b) : 19 heures après le repas. (1) : moyenne pour l'ensemble des prélèvements; $T$ : erreur standard moyenne.

FIG. 1A. - Influence de l'apport énergéfique en fin de gestation.

Log8: lots $H$; $\square$ : lots $B ; \star$ : lots $B$ significativement différents des lots $H(P \leqslant 0,050)$. FIG. 1B. - Influence de l'état des réserves corporelles.

: lots $G$; QZZZZ : lots $M$; * : lots $M$ significativement différents des lots $G(P \leqslant 0,050)$. 
L'absence de réserves corporelles suffisantes à l'approche du dernier tiers de la gestation n'entraîne pas de baisse significative du poids des agneaux à la naissance (fig. 1B), ni des rendements de fixation de l'énergie ef de l'azote (tabl. 1). La glycémie et la glutaminémie sont augmentées (fig. 1B), ce qui peut être dû à un accroissement de la synthèse de glucose à partir de l'acide propionique et du transport d'ammoniac du rumen sous la forme de glutamine, à la suite d'une élévation de l'activité fermentaire dans le rumen. L'histidinémie est diminuée sans doute à la suite d'une augmentation de la synthèse de protéines bactériennes dans le rumen. L'ensemble de ces phénomènes peut s'expliquer par une moindre diminution au cours de la fin de la gestation, du volume occupé par le tube digestif. Il y a aussi de légères baisses des teneurs sanguines de certains acides aminés non indispensables libres; en revanche, ni l'urémie, ni l'insulinémie ne sont modifiées.

\section{Chez les agneaux pendant le premier jour de la vie (fig. 2 A, 2 B).}

L'alimentation des brebis pendant la gestation n'a que peu d'effets sur les teneurs sanguines en acides aminés libres des agneaux; seules les teneurs en acides aminés non indispensables libres sont sensiblement plus faibles chez les agneaux nés de mères sous-alimentées en énergie. Chez ces derniers qui disposent de moindres réserves de glycogène, l'utilisation des acides aminés à des fins énergétiques est vraisemblablement accrue. Chez les agneaux à la naissance, comme chez les fœetus (Lemons ef al., 1976 ;
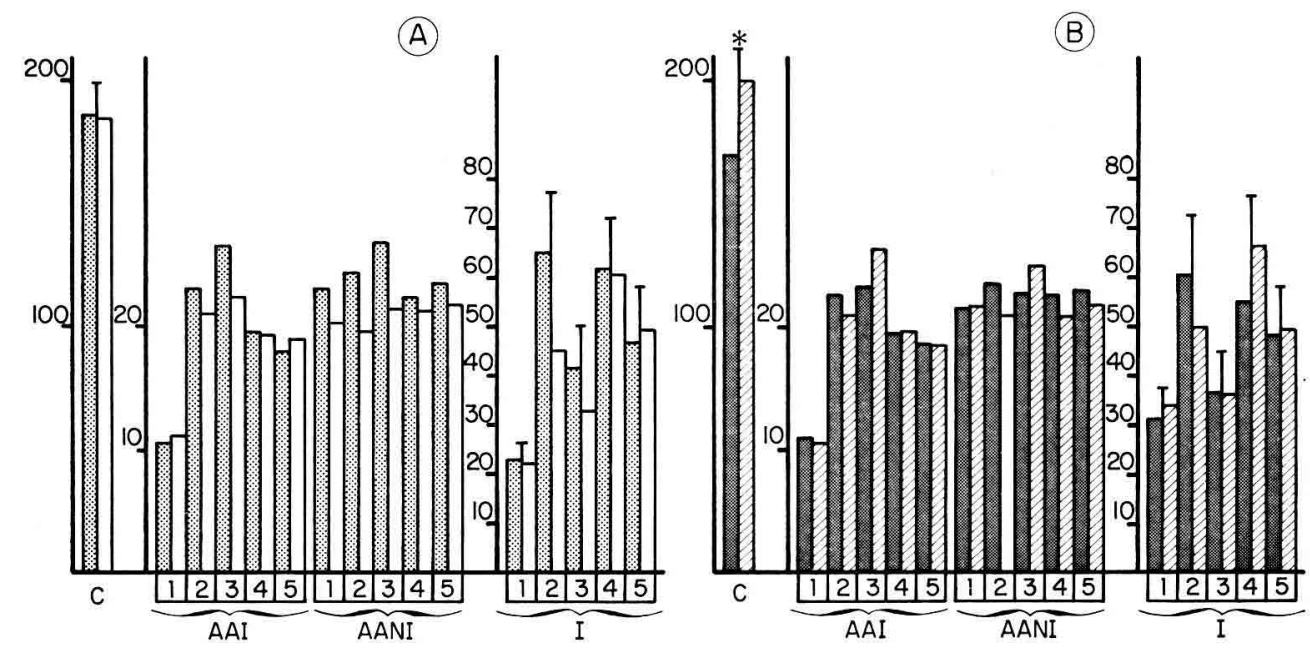

FIG. 2. - Insulinémie, teneurs en acides aminés libres du sang ef croissance des agneaux.

$\mathrm{I}$ : insuline ( $\mu \mathrm{U}$ p. $\mathrm{ml}$ de plasma) ; AAl : somme des teneurs en acides aminés indispensables ef semiindispensables (mg p. 100 de sang) ; AANI : somme des teneurs en acides aminés non indispensables (mg p. $100 \mathrm{~g}$ de sang); $\mathrm{C}:$ croissance des agneaux (g. p. j.). $1:$ naissance ; $2: 6 \mathrm{~h}$; $3: 24 \mathrm{~h} ; 4: 12$ jours ; $5: 24$ jours ; $T$ : erreur standard moyenne.

FIG. 2A. - Influence de l'apport énergétique en fin de gestation.

: lots $\mathrm{H}$; $\square$ : lots B.

FIG. 2B. - Influence de l'état des réserves corporelles des brebis.

: lots $G$; WUZD: lois $M$; * : lots $M$ significativement différents des lots $G(P \leqslant 0,050)$. 
Prior et Christenson, 1977 ; Smith et al., 1977), les teneurs sanguines de la plupart des acides aminés libres sont plus fortes que chez les brebis en fin de gestation. De la naissance à $24 \mathrm{~h}$, il y a de fortes augmentations des teneurs de tous les acides aminés indispensables et semi-indispensables libres; celles-ci peuvent être dues à une élévation de l'apport d'acides aminés à la fois par le catabolisme protéique et par la digestion du colostrum dans l'intestin. Le fait que les teneurs en acides aminés non indispensables libres varient peu, résulte de leur catabolisme élevé.

Il n'y a pas de différences significatives entre les insulinémies des quatre lots. Toutefois, l'insulinémie des agneaux est d'autant plus forte que la glycémie des brebis en fin de gestation est plus élevée, $19 \mathrm{~h}$ après le repas (coefficients de corrélation $R$ égaux à 0,38 et 0,34 respectivement aux âges de 6 et 24 h) ; ceci indique que la stimulation de la sécrétion d'insuline chez le fotus par le glucose circulant dans le sang de la brebis (Shelley, 1975), se manifeste après la naissance. A 24 h, la différence entre les insulinémies des deux agneaux nés de la même mère est d'autant plus importante que la différence entre leur poids de naissance est plus grande $(R=0,70)$. Ceci confirme le fait que l'insuline a une influence sur la croissance fœtale (Girard et al., 1976 ; Jost, 1977), en favorisant l'anabolisme. L'augmentation de l'insulinémie entre la naissance et $6 \mathrm{~h}$ est due à une augmentation de la sécrétion d'insuline sous l'effet des produits de la digestion (glucose, acides aminés indispensables), associés à l'accroissement de l'activité nerveuse parasympathique.

Chez les agneaux pendant la période d'allaitement (fig. 2 A, 2 B).

La sous-alimentation énergétique des brebis n'a aucun effet nolable chez les agneaux allaités. L'absence de réserves corporelles suffisantes à l'approche du dernier tiers de la gestation ne modifie ni les teneurs sanguines en acides aminés libres, ni l'insulinémie des agneaux ; en revanche, elle accroît significativement leur croissance. Ceci traduit l'accroissement de la production laitière des brebis.

\section{Conclusion.}

Le présent travail confirme que la restriction énergétique en fin de gestation des brebis portant plusieurs fơfus, n'entraîne pas de réduction de la croissance pondérale fœtale en automne, contrairement à ce qui se passe au printemps (Tissier et Thériez, 1979). Ceci semble résulter du fait qu'en automne, l'accroissement de la néoglucogenèse à partir des acides aminés provenant de la mobilisation des protéines corporelles, compense en grande partie le ralentissement de la néoglucogenèse à partir de l'acide propionique. En automne, comme au printemps (Grizard ef al., $1979 a$ et b), l'absence de réserves corporelles suffisantes à l'approche du dernier tiers de la gestation, stimule la production laitière pendant les 3 premières semaines de la lactation, ce qui favorise la croissance des agneaux.

La sécrétion d'insuline chez le fœtus est stimulée par la glycémie maternelle et semble favoriser la croissance pondérale fœtale. 


\section{Références}

CHRISTENSON R. K., PRIOR R. L., 1978. Uterine blood flow and nutrient uptake during late gestation in ewes with different number of fetuses. J. anim. Sci., 46, 189-200.

GIRARD J. R., CHANEZ C., KERVRAN A., TORDET-CARIDROIT C., ASSAN R., 1976. Studies on experimental hypotrophy in the rat. III. - Plasma insulin and glucagon. Biol. Neonate, 29, 262-265.

GRIZARD J., TISSIER M., CHAMPREDON C., PRUGNAUD J., PION R., 1979a. Variations des teneurs sanguines en acides aminés libres, urée et glucose chez la brebis en fin de gestation et début de lactation, influence de l'état nutritionnel en fin de gestation. Ann. Biol. anim. Bioch. Biophys., 19, 55-71.

GRIZARD J., TISSIER M., THERIEZ M., PION R., 1979b. Variations de l'insulinémie chez la brebis en fin de gestation et début de lactation, influence de l'état nutritionnel en fin de gestation. Ann. Biol. anim. Bioch. Biophys., 19, 73-78.

GRIZARD J., TISSIER M., PRUGNAUD J., PION R., 1977. Influence d'une restriction énergétique pendant la fin de la gestation sur l'insulinémie, la glycémie, l'urémie et l'aminoacidémie des brebis gestantes et allaitantes. C. R. Soc. Biol., 171, 1245-1253.

INRA, 1978. Alimentation des ruminants, Ed. INRA Publications (route de Saint-Cyr), 78000 Versailles. JOST A., 1977. Le rôle des hormones foetales dans la croissance des fœetus. J. Physiol. Paris, 73, 877-890.

LEMONS J. A., ADCOCK E. W., JONES M. D., NAUGHTON M. A., MESCHIA G., BATTAGLIA F. C., 1976. Umbilical uptake of aminoacids in the unstressed fetal lamb. J. clin. Invest., 58, 14281434.

MOORE S., SPACKMAN D. H., STEIN W. H., 1958. Chromatography of amino acids on sulfonated polystyrene resins. Analyt. Chem., 30, 1185-1190.

PAWLAK M., PION R., 1968. Influence de la supplémentation des protéines de blé par des doses croissantes de lysine sur la teneur en acides aminés libres du sang ef du muscle du rat en croissance. Ann. Biol. anim. Bioch. Biophys. 8, 517-530.

PRIOR R. L., CHRISTENSON R. K., 1976. Influence of dietary energy during gestation on lambing performance, and glucose metabolism in finn-cross ewes. J. anim. Sci., 43, 1114-1124.

PRIOR R. L., CHRISTENSON R. K., 1977. Gluconeogenesis from alanine in vivo by the ovine fetus and lamb. Am. J. Physiol., 233, E462-E468.

SHELLEY H. J., 1975. Insulin and the control of plasma glucose concentration in chronically catheterized foetal lambs. J. Physiol., 252, 66P-67P.

SMITH R. M., JARRET I. G., KING R. A., RUSSELL G. R., 1977. Amino acid nutrition of the fetal lamb. Biol. Neonate, 31, 305-310.

TISSIER M., THERIEZ M., 1979. Influence du niveau des apports énergétiques distribués à la brebis pendant la gestation sur le poids à la naissance et la croissance des agneaux. Ann. Biol. anim. Bioch. Biophys., 19, 235-240. 